\title{
The height of minimal Hilbert bases
}

\author{
Martin Henk and Robert Weismantel
}

\begin{abstract}
For an integral polyhedral cone $C=\operatorname{pos}\left\{a^{1}, \ldots, a^{m}\right\}, a^{i} \in \mathbb{Z}^{d}$, a subset $\mathcal{B}(C) \subset C \cap \mathbb{Z}^{d}$ is called a minimal Hilbert basis of $C$ iff (i) each element of $C \cap \mathbb{Z}^{d}$ can be written as a non-negative integral combination of elements of $\mathcal{B}(C)$ and (ii) $\mathcal{B}(C)$ has minimal cardinality with respect to all subsets of $C \cap \mathbb{Z}^{d}$ for which (i) holds. We give a tight bound for the so-called height of an element of the basis which improves on former results.
\end{abstract}

\section{Introduction}

Throughout the paper $\mathbb{R}^{d}$ denotes the $d$-dimensional Euclidean space, and pos $S$ the positive hull of a subset $S \subset \mathbb{R}^{d}$. The cardinality of a (finite) subset $S \subset \mathbb{R}^{d}$ is denoted by $\# S$ and the $i$-th unit vector is represented by $e^{i}$. A cone $C \subset \mathbb{R}^{d}$ is a set with the properties that $x+y \in C$ if $x, y \in C$ and $\lambda x \in C$ if $x \in C, \lambda \geq 0$. A cone $C$ is called pointed if the set $C \backslash\{0\}$ is strictly contained in an open halfspace, i.e., there exists $c \in \mathbb{R}^{d}$ such that $c^{T} x<0$ for all $x \in C \backslash\{0\}$. If $C=\operatorname{pos}\left\{a^{1}, \ldots, a^{m}\right\}$ with vectors $a^{i} \in \mathbb{R}^{d}, 1 \leq i \leq m$, then $C$ is called a polyhedral cone or a finitely generated cone.

Here we are studying integral polyhedral cones $C \subset \mathbb{R}^{d}$, i.e, there exist vectors $a^{i} \in \mathbb{Z}^{d} \backslash\{0\}$ for $1 \leq i \leq m$ such that $C=\operatorname{pos}\left\{a^{1}, \ldots, a^{m}\right\}$, or equivalently, $C=\left\{x \in \mathbb{R}^{d}: A x \leq 0\right\}$ for an appropriate matrix $A \in \mathbb{Z}^{n \times d}$.

From Gordan's lemma (cf. [1], [2]) we know that for every integral polyhedral cone $C$ there exists a set $\mathcal{B}(C) \subset C \cap \mathbb{Z}^{d}$ such that

1. each $z \in C \cap \mathbb{Z}^{d}$ can be expressed as a non-negative integral combination of elements in $\mathcal{B}(C)$, i.e., $z=\sum_{b \in \mathcal{B}(C)} z_{b} b, z_{b} \in \mathbb{N}$.

2. $\mathcal{B}(C)$ has minimal cardinality with respect to all subsets of $C \cap \mathbb{Z}^{d}$ for which (1) holds.

$\mathcal{B}(C)$ is called a minimal Hilbert basis of $C$. For short we just say basis of $C$. If $C$ is a pointed cone then $\mathcal{B}(C)$ is uniquely determined (cf. [3], [2]),

$$
\begin{array}{r}
\mathcal{B}(C)=\left\{b \in C \cap \mathbb{Z}^{d} \backslash\{0\}: b\right. \text { is not the sum of two other } \\
\text { vectors in } \left.C \cap \mathbb{Z}^{d} \backslash\{0\}\right\} .
\end{array}
$$

1991 Mathematics Subject Classification. Primary 52B20, 90C21; Secondary 11P21.

Key words and Phrases. Hilbert bases, rational polyhedral cones, integer programming. 
This implies that the basis $\mathcal{B}(C)$ is contained in the zonotope generated by $a^{1}, \ldots, a^{m}$. More precisely, we have

$$
\begin{aligned}
\mathcal{B}(C) & \subset\left\{a^{1}, \ldots, a^{m}\right\} \cup \\
& \left\{a \in C \cap \mathbb{Z}^{d} \backslash\{0\}: a=\sum_{i=1}^{m} \lambda_{i} a^{i}, 0 \leq \lambda_{i}<1,1 \leq i \leq m\right\} .
\end{aligned}
$$

We want to remark that (minimal) Hilbert bases occur under many different names in various fields of mathematics such as integer programming (cf. [2], [4], [5], [6]) or in the context of special desingularizations of toric varieties (cf. [7], [8], [9],[10]). However, very little is known about the geometrical structure of the basis elements. Here we focus on the height of the Hilbert basis.

Definition 1. Let $C=\operatorname{pos}\left\{a^{1}, \ldots, a^{m}\right\}, a^{i} \in \mathbb{Z}^{d}$, be a pointed cone. For $b \in \mathcal{B}(C)$ the number

$$
h_{C}(b):=\min \left\{\sum_{i=1}^{m} \lambda_{i}: b=\sum_{i=1}^{m} \lambda_{i} a^{i}, \lambda_{i} \geq 0,1 \leq i \leq m\right\}
$$

is called the height of the basis element $b$.

It is straightforward to see that for dimension 2 the height is not greater than 1 and from Caratheodory's theorem and (1.2) one easily derives the bound $h_{C}(b)<d, b \in \mathcal{B}(C), C \subset \mathbb{R}^{d}$. Indeed, it was proven by Ewald \& Wessels [11] that

$$
h_{C}(b)<d-1, \quad b \in \mathcal{B}(C), \quad d \geq 3,
$$

is an asymptotically tight upper bound for the height (see also [12]). Here we sharpen the bound in the following way.

Theorem 1. Let $C=\operatorname{pos}\left\{a^{1}, \ldots, a^{m}\right\}, a^{i} \in \mathbb{Z}^{d}$, be a d-dimensional pointed cone. For $b \in \mathcal{B}(C)$ one has

$$
h_{C}(b) \leq(d-1)-\frac{d-2}{\left|\operatorname{det}\left(a^{i_{1}}, \ldots, a^{i_{d}}\right)\right|},
$$

where $\left\{a^{i_{1}}, \ldots, a^{i_{d}}\right\} \subset\left\{a^{1}, \ldots, a^{m}\right\}$ is a subset of d linearly independent integral points such that $b \in \operatorname{pos}\left\{a^{i_{1}}, \ldots, a^{i_{d}}\right\}$.

This bound is tight for various families of cones. For example, let $r \in \mathbb{N} \backslash\{0\}$ and let (cf. [11])

$$
C_{r}^{d}=\operatorname{pos}\left\{e^{1}, \ldots, e^{d-1}, r e^{d}+\sum_{i=1}^{d-1} e^{i}\right\}
$$

The point $b=(1, \ldots, 1)^{T}$ is an element of the basis with

$$
h_{C}(b)=(d-1) \cdot \frac{r-1}{r}+\frac{1}{r}=d-1-\frac{d-2}{\left|\operatorname{det}\left(e^{1}, \ldots, e^{d-1}, r e^{d}+\sum_{i=1}^{d-1} e^{i}\right)\right|} .
$$

\section{Proof of Theorem 1}

The proof is prepared by the next two simple lemmas. 
Lemma 1. Let $p, r \in \mathbb{N}$ such that $1 \leq p \leq r-1$. We define $\mathcal{M}(p, r)=\{j \in\{0, \ldots, r-1\}$ : $(j \cdot p) \bmod r \leq p\}$. Then $\# \mathcal{M}(p, r)=p+\operatorname{gcd}(p, r)$.

Proof. Obviously, if $\operatorname{gcd}(p, r)=1$ then $\{(j \cdot p) \bmod r: 0 \leq j \leq r-1\}=\{0, \ldots, r-1\}$ and the statement is true. Hence $\# \mathcal{M}(p / q, r / q)=p / q+1$ where $q=\operatorname{gcd}(p, r)$. Since

$$
\left(j+i \cdot \frac{r}{q}\right) \cdot p \bmod r=q \cdot\left(j \cdot \frac{p}{q} \bmod \frac{r}{q}\right), 0 \leq j \leq \frac{r}{q}-1,0 \leq i \leq q-1,
$$

we get $\# \mathcal{M}(p, r)=q \cdot \# \mathcal{M}(p / q, r / q)=p+\operatorname{gcd}(p, r)$.

The next lemma is quite obvious and can easily be proved by induction on the number $n$.

Lemma 2. Let $m, n$ be positive integers and let $\mathcal{N}_{i} \subset\{1, \ldots, n\}$ for $1 \leq i \leq m$. If $\sum_{i=1}^{m} \# \mathcal{N}_{i} \geq$ $(m-1) \cdot n+k, k \in\{1, \ldots, n\}$, then $\#\left(\bigcap_{i=1}^{m} \mathcal{N}_{i}\right) \geq k$.

We are now ready for the proof of Theorem 1.

Proof of Theorem 1. Let $b \in \mathcal{B}(C)$ and w.l.o.g. let $\left\{a^{1}, \ldots, a^{d}\right\}$ be a subset of the generators $a^{1}, \ldots, a^{m}$ of the cone $C$ such that $a^{1}, \ldots, a^{d}$ are linearly independent and $b \in \operatorname{pos}\left\{a^{1}, \ldots, a^{d}\right\}$. Let $\Lambda \subset \mathbb{Z}^{d}$ be the lattice generated by $\left\{a^{1}, \ldots, a^{d}, b\right\}$, $\operatorname{det}(\Lambda)$ its determinant and

$$
r=\left|\operatorname{det}\left(a^{1}, \ldots, a^{d}\right)\right| / \operatorname{det}(\Lambda) \in \mathbb{N}
$$

be the index of the sublattice generated by $a^{1}, \ldots, a^{d}$ w.r.t. $\Lambda$ (cf. [13]). In the following we show

$$
h_{C}(b) \leq(d-1)-(d-2) \frac{\operatorname{det}(\Lambda)}{\left|\operatorname{det}\left(a^{1}, \ldots, a^{d}\right)\right|}=(d-1)-\frac{d-2}{r},
$$

which is a slightly stronger inequality as posed in Theorem 1. To this end let $b \notin\left\{a^{1}, \ldots, a^{d}\right\}$. Furthermore, since $b$ is also contained in the minimal Hilbert basis of the cone $\operatorname{pos}\left\{a^{1}, \ldots, a^{d}\right\}$ we may assume by (1.2) that $b=\sum_{i=1}^{d} \lambda_{i} a^{i}$ with $0 \leq \lambda_{i}<1$. It is quite easy to see that the coefficients $\lambda_{i}$ have a representation as

$$
\lambda_{i}=\frac{p_{i}}{r}, \quad p_{i} \in\{0, \ldots, r-1\}, 1 \leq i \leq d,
$$

with $\operatorname{gcd}\left(p_{1}, \ldots, p_{d}, r\right)=1$ and that

$$
\left\{\sum_{i=1}^{d}\left(\frac{\left(j \cdot p_{i}\right) \bmod r}{r}\right) a^{i}: 1 \leq j \leq r-1\right\} \subset C \cap \mathbb{Z}^{d} \backslash\{0\} .
$$

Now, by definition we have $h_{C}(b) \leq \sum_{i=1}^{d}\left(p_{i} / r\right)$ and thus it suffices to show $\sum_{i=1}^{d} \frac{p_{i}}{r} \leq(d-1)-$ $(d-2) / r$ (cf. (2.1)). Assume the contrary, i.e.,

$$
\sum_{i=1}^{d} p_{i} \geq(d-1)(r-1)+2
$$

Then $r \geq 3$ and we show that $b$ can be written as the sum of two elements contained in the set on the left hand side of (2.2). This contradicts (1.1).

For $1 \leq i \leq d$ let

$$
\mathcal{M}\left(p_{i}, r\right)=\left\{j \in\{0, \ldots, r-1\}: j p_{i} \bmod r \leq p_{i}\right\} .
$$


Lemma 1 yields the bound $\# \mathcal{M}\left(p_{i}, r\right) \geq p_{i}+1$ and by $(2.3)$ we get

$$
\sum_{i=1}^{d} \# \mathcal{M}\left(p_{i}, r\right) \geq(d-1) r+3
$$

Lemma 2 says that the intersection $\cap_{i=1}^{d} \mathcal{M}\left(p_{i}, r\right)$ contains an element $k \in\{2, \ldots, r-1\}$, say. By the definition of the sets $\mathcal{M}\left(p_{i}, r\right)$ we have

$$
p_{i}=\left(\left(k \cdot p_{i}\right) \bmod r\right)+\left(\left((r-1-k) \cdot p_{i}\right) \bmod r\right), \quad 1 \leq i \leq d,
$$

and we get the desired contradiction

$$
b=\sum_{i=1}^{d}\left(\frac{\left(k \cdot p_{i}\right) \bmod r}{r}\right) a^{i}+\sum_{i=1}^{d}\left(\frac{\left((r-1-k) \cdot p_{i}\right) \bmod r}{r}\right) a^{i} .
$$

\section{Some consequences of Theorem 1}

Theorem 1 may be used to derive the following lower bound.

Corollary 1. Let $C=\operatorname{pos}\left\{a^{1}, \ldots, a^{d}\right\}$ be a pointed cone such that $a^{1}, \ldots, a^{d} \in \mathbb{Z}^{d}$ are linearly independent. If $\mathcal{B}(C)=\left\{a^{1}, \ldots, a^{d}\right\} \cup\left\{z \in \mathbb{Z}^{d}: z=\sum_{i=1}^{d} \lambda_{i} a^{i}, 0 \leq \lambda_{i}<1\right\}$ then for $b \in \mathcal{B}(C)$ contained in the interior of $C$ one has

$$
h_{C}(b) \geq 1+\frac{d-2}{\left|\operatorname{det}\left(a^{1}, \ldots, a^{d}\right)\right|} .
$$

Proof. Since $b$ is contained in the interior of $C$ the lattice point $\bar{b}=\sum_{i=1}^{d} a^{i}-b$ is contained in the half open parallelepiped generated by $a^{1}, \ldots, a^{d}$. Hence, by assumption $\bar{b} \in \mathcal{B}(C)$ and Theorem 1 yields

$$
h_{C}(\bar{b})=d-h_{C}(b) \geq 1+(d-2) /\left|\operatorname{det}\left(a^{1}, \ldots, a^{d}\right)\right| .
$$

The next corollary shows an application of Theorem 1 in integer programming.

Corollary 2. Let $A \in \mathbb{Z}^{m \times d}$ with all subdeterminants at most $\alpha$ in absolute value and let $b \in \mathbb{Z}^{m}$, $c \in \mathbb{Z}^{d}$ be given vectors. If $\widetilde{z}$ is a feasible, non-optimal solution of the program $\max \left\{c^{T} z: A z \leq\right.$ $\left.b, z \in \mathbb{Z}^{d}\right\}$, then there exists a feasible solution $\widehat{z}$ such that $c^{T} \widehat{z}>c^{T} \widetilde{z}$ and

$$
|\widehat{z}-\widetilde{z}|_{\infty} \leq(d-1) \alpha-\frac{d-2}{d^{d / 2} \alpha^{d-2}},
$$

where $|\cdot|_{\infty}$ denotes the maximum norm.

Proof. Let $z^{*}$ be a feasible solution with $c^{T} z^{*}>c^{T} \widetilde{z}$. We split the system $A x \leq b$ into subsystems $A_{1} x \leq b_{1}, A_{2} x \leq b_{2}$ such that $A_{1} \widetilde{z} \leq A_{1} z^{*}$ and $A_{2} \widetilde{z} \geq A_{2} z^{*}$. Let $C$ be the cone

$$
C=\left\{x \in \mathbb{R}^{d}: A_{1} x \leq 0, A_{2} x \geq 0\right\}
$$


and $w^{1}, \ldots, w^{n} \in \mathbb{Z}^{d}$ such that $C=\operatorname{pos}\left\{w^{1}, \ldots, w^{n}\right\}$. Using Cramer's rule we obtain that $\left|w^{j}\right|_{\infty} \leq$ $\alpha, 1 \leq j \leq n$. Since $z^{*}-\widetilde{z} \in C$ there exist $l \leq d$ linearly independent vectors $w^{i_{1}}, \ldots, w^{i_{l}}$ such that $z^{*}-\widetilde{z} \in \bar{C}=\operatorname{pos}\left\{w^{i_{1}}, \ldots, w^{i_{l}}\right\}$. It follows that

$$
z^{*}-\widetilde{z}=\sum_{i=1}^{k} n_{i} b^{i}, \quad n_{i} \in \mathbb{N} \backslash\{0\}
$$

for some $b^{1}, \ldots, b^{k} \in \mathcal{B}(\bar{C})$. It is easy to see that $\widetilde{z}+b^{i}, i \in\{1, \ldots, k\}$, is a feasible solution. On account of the condition $c^{T} z^{*}>c^{T} \widetilde{z}$ we may assume that $c^{T}\left(\widetilde{z}+b^{1}\right)>c^{T} \widetilde{z}$. We define $\widehat{z}:=\widetilde{z}+b^{1}$ and write $b^{1}$ as

$$
b^{1}=\sum_{j=1}^{l} \lambda_{j} w^{i_{j}}
$$

with $\lambda_{1}, \ldots, \lambda_{l} \geq 0$. Applying Theorem 1 to the $l$-dimensional cone $\bar{C}$ together with the Hadamard inequality gives

$$
|\widehat{z}-\widetilde{z}|_{\infty} \leq \alpha\left(\sum_{j=1}^{l} \lambda_{j}\right) \leq \alpha\left((l-1)-\frac{l-2}{\left.\left|w^{i_{1}}\right| \cdots\left|w^{i_{l}}\right|\right)}\right)
$$

As $\left|w^{i_{j}}\right| \leq d^{1 / 2} \alpha$, we obtain

$$
|\widehat{z}-\widetilde{z}|_{\infty} \leq(l-1) \alpha-\frac{l-2}{d^{l / 2} \alpha^{l-1}} \leq(d-1) \alpha-\frac{d-2}{d^{d / 2} \alpha^{d-1}},
$$

where the last inequality can be verified with elementary algebra.

We remark that the bound of Corollary 2 strengthens the bound of $d \alpha$ given in [14].

We are grateful to Les Trotter jr. and Günter M. Ziegler for helpful discussions and comments.

\section{References}

[1] P. Gordan, Über die Auflösung linearer Gleichungen mit reellen Coefficienten, Math. Ann. 6 (1873), $23-28$.

[2] A. Schrijver, Theory of linear and integer programming, John Wiley and Sons, Chichester, 1986.

[3] J.G. van der Corput, Über Systeme von linear-homogenen Gleichungen und Ungleichungen, Proceedings Koninklijke Akademie van Wetenschappen te Amsterdam 34 (1931), 368-371.

[4] A. Sebö, Hilbert bases, Caratheodory's Theorem and combinatorial optimization, Proc. of the IPCO conference, Waterloo, Canada, 1990, 431-455.

[5] R. Urbaniak, R. Weismantel, and G.M. Ziegler, A variant of Buchberger's algorithm for integer programming, SIAM J. Discrete Math., to appear.

[6] R. Weismantel, Hilbert bases and the facets of special knapsack polytopes, Math. Oper. Res. 21(4) (1996), 896-904.

[7] C. Bouvier and G. Gonzalez-Springberg, G-désingularisations de variétés toriques, C. R. Acad.Sci. Paris, t.315, Série I, (1992), 817-820. 
[8] D. Dais, M. Henk, and G.M. Ziegler, On the existence of crepant resolutions of Gorenstein abelian quotient singularities in dimensions $\geq 4$, in preparation (1996).

[9] G. Ewald, Combinatorial Convexity and Algebraic Geometry, Graduate Texts in Mathematics Vol. 168, Springer, Berlin, 1996.

[10] T. Oda, Convex bodies and algebraic geometry, Springer, New-York, 1985.

[11] G. Ewald and U. Wessels, On the ampleness of invertible sheaves in complete projective toric varieties, Result. Math. 19 (1991), 275-278.

[12] J. Liu, L.E. Trotter, Jr., and G.M. Ziegler, On the Height of the minimal Hilbert basis, Result. Math. 23 (1993), 374-376.

[13] P.M. Gruber and C.G. Lekkerkerker, Geometry of numbers, 2nd ed., North-Holland, Amsterdam, 1987.

[14] W. Cook, A.M.H. Gerards, A. Schrijver, and É. Tardos, Sensitivity theorems in integer programming problems, Math. Programming 34 (1986), 63-70.

Martin Henk, Robert Weismantel

Konrad-Zuse-Zentrum für Informationstechnik (ZIB) Berlin

Takustraße 7

D-14195 Berlin-Dahlem, Germany

E-mail: henk@zib.de, weismantel@zib.de

Eingegangen am 30. April 1997. 